\title{
The TMT Laser Guide Star Facility
}

\author{
Corinne Boyer $^{1, a}$, Brent Ellerbroek, Luc Gilles, and Lianqi Wang \\ ${ }^{1}$ TMT Observatory Corporation, 2632 E. Washington Blvd, Pasadena CA, USA
}

\begin{abstract}
The Laser Guide Star Facility (LGSF) system of the Thirty Meter Telescope (TMT) will generate the artificial laser guide stars required by the TMT Laser Guide Star AO systems. The LGSF will use multiple sodium lasers to generate and project several asterisms from a laser launch telescope located behind the TMT secondary mirror. In particular, the LGSF will generate a 6-guide-star asterism for the TMT Narrow Field Infrared Adaptive Optics System (NFIRAOS). In this paper, we will present the LGSF requirements, system level tradeoffs, expected performance and the current status of the design, which is based upon solid state continuous wave, $589 \mathrm{~nm}$ lasers and conventional beam transport optics.
\end{abstract}

\section{Introduction}

The TMT science goals [1] require the achievement of near diffraction-limited performance in the nearand mid-InfraRed through the use of AO systems specifically optimized for the TMT instrumentation. These goals translate into the following specific requirements which are relevant to the LGSF: (i) high sky coverage, which implies Laser Guide Star (LGS) AO for higher-order wavefront correction, and (ii) diffraction-limited wavefront compensation over the large TMT aperture, which requires multiple sodium LGS and tomographic wavefront reconstruction to defeat the cone effect.

TMT has defined a suite of first decade instruments that will implement different AO techniques and will require up to 4 different LGS asterisms with various number of LGS and different geometries. Even though not all these instruments will be implemented at first light, the LGSF will be designed to generate the asterisms of all planned TMT AO systems. These asterisms are described below:

- The Multi Conjugate AO (MCAO) asterism, which consists of 6 LGS, 5 LGS equally spaced on a circle of radius of 35 arcsec and one additional on-axis LGS. This asterism will be used by the first light Narrow Field Infrared Adaptive Optics System (NFIRAOS) [2], [3], which will feed up to 3 narrow field, near IR instruments for spectroscopy and imaging.

- The Laser Tomography AO (LTAO) asterism, which consists of 3 LGS, equally spaced on a circle of radius of 70 arcsec. This asterism is dedicated to instruments for narrow field mid IR spectroscopy and imaging.

- The Multi Object AO (MOAO) asterism, which consists of 8 LGS, 3 LGS equally spaced on a circle of radius of 70 arcsec and 5 LGS equally spaced on a circle of radius of 150 arcsec. This asterism is dedicated to moderate field, near IR multi-object IFU spectroscopy.

- The Ground Layer AO (GLAO) asterism, which consists of 5 LGS, 4 LGS equally spaced on a circle of radius of 510 arcsec and one additional on-axis LGS. This asterism is dedicated to wide field optical spectroscopy.

The LGSF will be also designed to generate any additional asterism with up to 9 LGS and with a radius varying from to 5 to 510 arcsec.

The purpose of this paper is to describe the decisions made regarding key component technologies and high-level design options (section 2), and to present the TMT LGSF requirements and associated trade studies (section 3). Section 4 then presents the current status of the TMT LGSF conceptual design.

\section{Technology and design choices}

In order to lower the risks and maximize the science utility at first light, TMT has taken a conservative approach for the LGSF critical components needed at first light and the top-level design choices:

\footnotetext{
${ }^{\text {a }}$ e-mail: cboyer@tmt.org
} 
Fig. 1. LGSF Conceptual Design

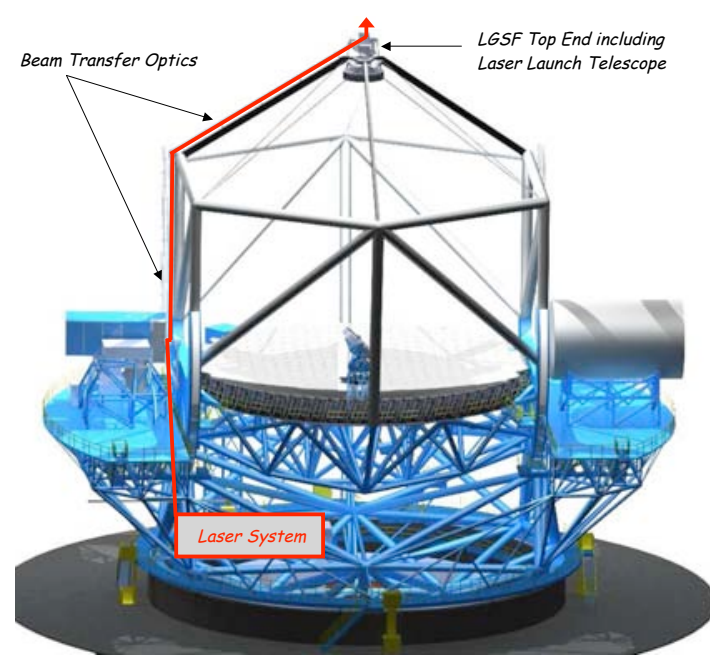

- The laser system will implement 589nm solid-state lasers, with either a continuous wave (CW) or mode locked CW pulse format, even though guide star elongation is a very signficant issue for a 30meter telescope ( 4 arcsec) and the required guidestar brightness consequently increases by about a factor of 2.5 in comparison with the current generation of 8-10 meter telescopes. Such lasers have already been demonstrated at the TMT power level requirements using sum-frequency-generation technology [4]. Other laser technologies such as $\mu$ s-pulsed lasers with dynamic refocusing (to eliminate the elongation issue and consequently to reduce the laser power requirement), or ms-pulsed lasers (to eliminate the fratricide impact of Rayleigh backscatter interference between separate beacons, and consequently improve the AO performance) are not currently available at/or near the power level required for TMT.

- The laser launch telescope will be located behind the TMT secondary mirror (center-launched design) to minimize guidestar elongation and therefore reduce the complexity of the LGS WFS detectors. The option to launch the beams from several laser launch telescopes located around the edge of the primary mirror (side-launched design) has been rejected because of the elongation, which is doubled with this concept ( 8 arcsec).

- The laser system is mounted within the azimuth structure to allow the lasers to operate in a fixed gravity orientation. In addition, wind obstruction on the telescope and vibrations coupled into the telescope structure are reduced with such a location and access to the laser system is facilitated. However, a longer and more complex beam path is needed to transport the laser beams onto the telescope center section and then to the launch telescope.

- The LGSF will incorporate conventional optics to transport the beams from the laser system to the laser launch telescope. Although progress in the hollow core fiber technology is encouraging, it is not currently an option for the TMT path length and the TMT peak power requirements, especially at first light. Conventional-optics-based beam transport systems have been developed and implemented in several astronomical facilities and are operated successfully on a day-to-day basis [5].

The LGSF current design approach is illustrated in Figure 1. It consists of three main sub-systems: (1) the laser system located within the telescope azimuth structure, (2) the beam transfer optics, which transports the beams from the telescope azimuth structure to the telescope top end and (3) the LGSF top end, which consists of the asterism generator and the laser launch telescope. The LGSF also includes the Laser Safety System, dedicated for the protection of people, observatory hardware, aircrafts, satellites and neighboring telescopes. The conceptual design was developed in 2006 by NOAO in collaboration with the TMT project office [6], and was updated in 2008 to adapt for the new Ritchey-Chrétien optical design of the TMT and to compensate for the TMT telescope top end flexure [7].

The LGSF design approach may be revisited during the next phase of the design, in particular the decisions regarding the laser system and/or laser launch system locations will be scrutinized again based upon (i) additional simulations comparing the center-launched and side-launched designs in 
Corinne Boyer et al.: The TMT Laser Guide Star Facility

Table 1. LGSF Requirements

\begin{tabular}{ll}
\hline Parameter & Requirement \\
\hline LGS Power & 25W per LGS (150W in 6 LGS at first light) \\
LGS Beam Quality & 85\% of energy in a 1.2 x diffraction-limited core \\
LGS Tip/Tilt Jitter & Tip/Tilt jitter $<50$ mas (1-axis, 1-sigma) \\
LGS Polarization & $98 \%$ circularly polarized \\
LGS Throughput & $75 \%$ \\
Asterisms & Rapid switching from one asterism to another \\
LGS Pointing & Blind pointing accuracy on the sky of $\sim 1$ arcsec \\
LGS Operation & Downtime $<0.5 \%$ \\
\hline
\end{tabular}

Table 2. Laser System Requirements

\begin{tabular}{ll}
\hline Parameter & Requirement \\
\hline Laser Power & $6 \times 25 \mathrm{~W} \mathrm{CW}$ or mode locked CW solid state lasers for first light AO system \\
Sodium Layer Coupling & 130 photons $\mathrm{m}^{2} / \mathrm{s} / \mathrm{W} /$ ion \\
Beam Quality & $95 \%$ of energy in a $1.1 \mathrm{x}$ diffraction-limited core \\
Polarization & $>98 \%$ linearly polarized \\
Power Stability & $3 \%[15 \%]$ short $[$ long $]$ term \\
Output Beam Diameter & $5 \mathrm{~mm} \pm 0.3 \mathrm{~mm}$ \\
& Pointing jitter $\leq 3 \mu \mathrm{rad}$, long term drift $\leq 100 \mu \mathrm{rad}$ and lateral shift $\leq 5 \mu \mathrm{mm}$ \\
Wavelength Control & Tune on/off D2 line \\
Laser Operation & Downtime $<0.85 \%$ (yields $\sim 1400$ hours MTBF) \\
Laser System Upgrade & Additional lasers for MOAO asterism \\
& Increased power for second decade of TMT instruments \\
\hline
\end{tabular}

terms of AO performance (i.e. the wavefront error due to LGS WFS measurement noise error), (ii) progress towards manufacturing smaller, lighter and more robust lasers with designs compatible with changing gravity orientation, (iii) the feasibility to develop bigger LGS WFS detectors and (iv) improvements in hollow core fiber technology.

\section{LGSF requirements}

The top-level requirements for the TMT LGSF and for the TMT laser system are summarized in Table 1 and Table 2. TMT has carried out a detailed system engineering process to define these requirements in particularly in the areas of required LGS power, laser launch telescope diameter, LGS beam quality, and LGS pointing jitter. The following sub-sections describe the results of these trade studies.

\subsection{LGS power requirement}

The LGS power requirement is derived from the LGS WFS signal requirement, which is estimated from simulations including the LGS elongation, the WFS spot size, WFS detector performance, and the processing algorithms used both for the LGS gradient estimation and LGS tomography. The dependence of the LGS WFS signal level upon guidestar laser power and several other parameters has been modeled using the formula:

$$
N_{p d e}=\left(P_{L} T_{L G S F} T_{B Q}\right)\left(s C_{s} \sec \theta\right)\left(T_{A T M}^{2 \sec \theta} /(z \sec \theta)^{2}\right)\left(A T_{A O S} \tau\right)
$$

The definitions and values of the parameters appearing in equation (1) are summarized in Table 3. The formula is an oversimplification of the reality, since it neglects the impact of sodium layer saturation and the spectral characteristics, but still provides a reasonable match with existing solid state, CW lasers. The required LGS power depends then upon the assumptions made for (i) the sodium layer parameters including the integrated column density $C_{s}$ of the sodium ions and the coupling efficiency of the sodium layer $s$ and (ii) the uplink/downlink optical throughput and atmospheric transmittance 
First conference on Adaptive Optics for Extremely Large Telescopes

Table 3. LGS return signal for nominal and pessimistic cases

\begin{tabular}{|c|c|c|c|c|}
\hline \multirow[t]{2}{*}{ Definition } & \multicolumn{2}{|c|}{ Pessimistic Throughput } & \multicolumn{2}{|c|}{ Nominal Throughput } \\
\hline & $C_{s}$ reduced & $C_{s}$ specified & $C_{s}$ reduced & $C_{s}$ specified \\
\hline A: WFS sub-aperture area, $\mathrm{m}^{2}$ & 0.25 & 0.25 & 0.25 & 0.25 \\
\hline$C_{s}:$ Column density, ions $/ \mathrm{m}^{2}$ & $3 \times 10^{13}$ & $4 \times 10^{13}$ & $3 \times 10^{13}$ & $4 \times 10^{13}$ \\
\hline$P_{L}:$ Average laser power, $\mathrm{W}$ & 25 & 25 & 25 & 25 \\
\hline $\begin{array}{l}s: \text { Unsaturated coupling efficiency, } \\
\text { photons } \mathrm{m}^{2} / \mathrm{s} / \mathrm{W} / \text { ion }\end{array}$ & 130 & 130 & 130 & 130 \\
\hline $\begin{array}{l}T_{A O S}: \text { End-to-end telescope/AO efficiency, } \\
\text { photoelectrons/photon }\end{array}$ & 0.34 & 0.34 & 0.42 & 0.42 \\
\hline$T_{A T M}:$ Atmospheric transmittance at zenith & 0.7 & 0.7 & 0.84 & 0.84 \\
\hline$T_{L G S F}:$ End-to-end LGSF transmittance & 0.75 & 0.75 & 0.75 & 0.75 \\
\hline$T_{B Q}$ : Beam quality equivalent transmittance & 0.8 & 0.8 & 0.8 & 0.8 \\
\hline$\tau:$ WFS integration time, sec & 0.00125 & 0.00125 & 0.00125 & 0.00125 \\
\hline$\theta$ : Zenith angles, degrees & 0 & 0 & 0 & 0 \\
\hline$z:$ Sodium layer range, $\mathrm{km}$ & 91.8 & 91.8 & 89.4 & 89.4 \\
\hline$N_{p d e}:$ PDEs/sub-aperture/frame & 360 & 480 & 680 & 900 \\
\hline
\end{tabular}

Table 4. Impact of reduced LGS WFS signal level on AO performance (Inc.: Incremental, Op.: Optimal)

\begin{tabular}{cccc}
\hline $\begin{array}{c}\text { Signal level at } 800 \mathrm{~Hz} \\
\text { PDEs }\end{array}$ & $\begin{array}{c}\text { Inc. WFE at } 800 \mathrm{~Hz} \\
\mathrm{~nm}\end{array}$ & $\begin{array}{c}\text { Op. AO frame rate } \\
\mathrm{Hz}\end{array}$ & $\begin{array}{c}\text { Inc. WFE at op. frame rate } \\
\mathrm{nm}\end{array}$ \\
\hline 900 & 0 (baseline) & 800 & - \\
500 & 47 & 600 & 43 \\
250 & 91 & 400 & 75 \\
\hline
\end{tabular}

$T_{A T M}$. For all these parameters, conservative design values have been used to maximize the availability of the LGS AO for TMT.

A LGS WFS signal requirement of 900 photodetection events per sub-aperture and per frame (corresponding to a signal of $2.810^{6} \mathrm{PDEs} / \mathrm{m}^{2} / \mathrm{sec}$ and a RMS wavefront error (WFE) due to noise at zenith of $43 \mathrm{~nm}$ ) can be met by using approximately $25 \mathrm{~W}$ of laser power per laser guidestar. Photon return will vary with conditions as described in Table 3, but the impact on AO performance due to reduction in photon return can be minimized by optimizing the $\mathrm{AO}$ frame rate (see Table 4).

\subsection{Laser Launch Telescope aperture diameter and beam profile}

Several laser guide star AO systems operating on $8-10 \mathrm{~m}$ class telescopes utilize launch telescopes approximately $0.5 \mathrm{~m}$ in diameter and project Gaussian beams to the sky. We have conducted several trade studies to verify if these design choices apply to the LGSF of TMT. These simulations allow quantifying how relative the LGS WFS signal requirements and consequently the LGS power requirements will vary with the above parameters. The results of these studies are summarized in this paper:

- A Gaussian laser beam profile with a $1 / \mathrm{e}^{2}$ beam diameter of 0.6 times the laser launch telescope diameter is preferable to a top-hat beam profile.

- Increasing the launch telescope diameter beyond $0.5 \mathrm{~m}$ is not cost-effective, as even a diameter as large as $1.0 \mathrm{~m}$ reduces the laser power requirement by only $5-10 \%$.

- Our most recent simulations, performed for different turbulence conditions $(r 0)$, sodium layer thicknesses and LGS signal levels, show that reducing the launch telescope diameter further to $0.4 \mathrm{~m}$ increases the RMS wavefront error only slightly $(\sim 13 \mathrm{~nm})$ for the case of elongated LGS, and that the impact upon performance with dynamic refocusing is negligible. Adjusting the launch telescope focus to track the range of the sodium layer for each science observation provides a fairly small but not entirely negligible improvement $(<3 \%)$ in LGS spot size. This feature is included in the design, since it will also simplify the initial installation and commissioning of the LGSF. 


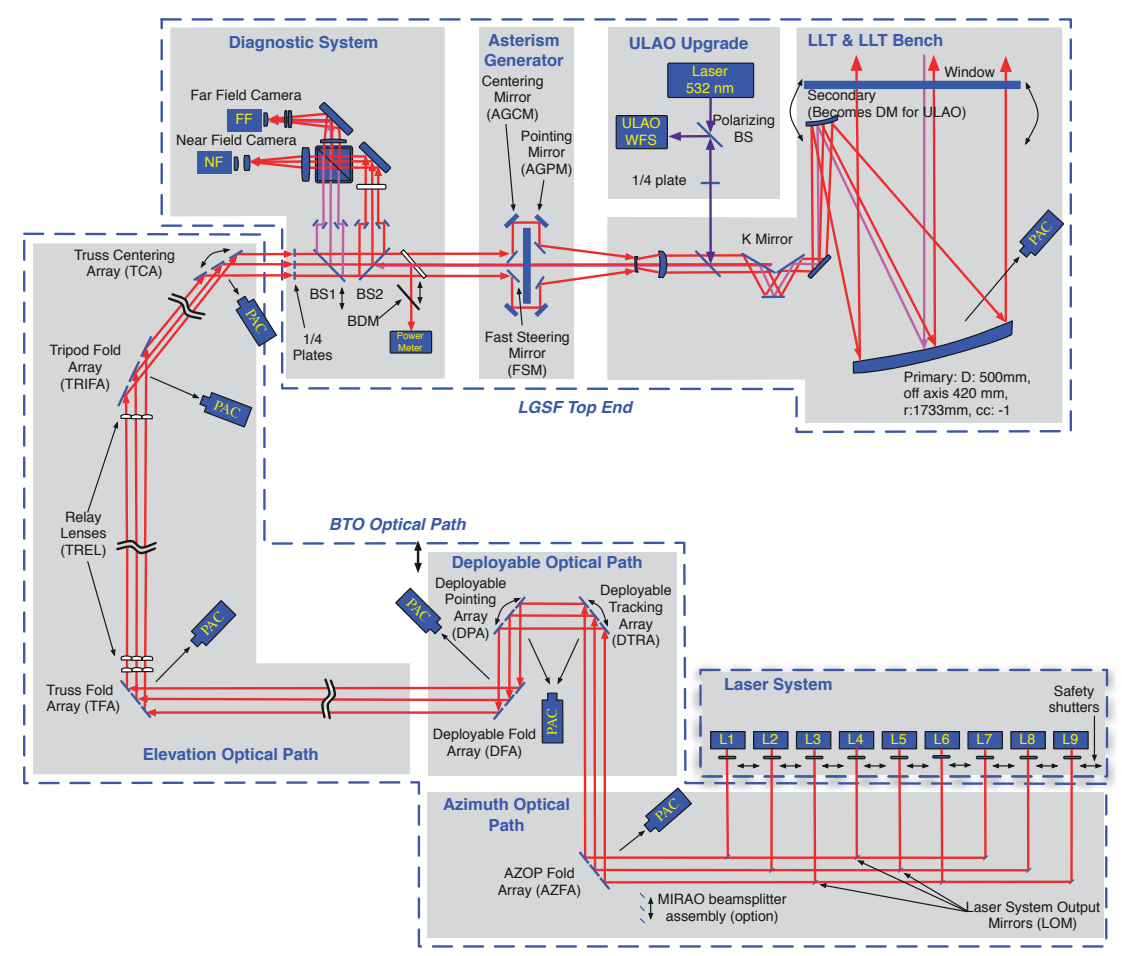

Fig. 2. LGSF Optical Schematic

\subsection{LGS pointing jitter}

The budget for the LGS pointing accuracy (tip/tilt jitter) is approximately 50 mas on the sky, given (i) the expected linear dynamic range of the matched filter algorithm used for estimating the LGS centroids and (ii) the laser launch telescope diameter. The most critical term in this budget is the term for atmospheric tip/tilt servo lag of 40 mas.

To null the errors as measured by the LGS WFS, the LGS pointing jitter will be controlled by fast steering mirrors (FSM). Two locations have been studied for these mirrors: (1) within the LGSF optical path and (2) just before the LGS WFS in each AO system. In both cases, the bandwidth of the pointing loop is limited by the finite sampling rate of the LGS WFS $(800 \mathrm{~Hz})$. In the LGSF implementation case, the bandwidth is also limited by the round-trip time-of-flight delay to the sodium layer. Although the correction is more efficient when the FSM is located within the AO system, the implementation within the LGSF is less expensive and still meets the 50 mas budget requirement (Simulated tip/tilt residual jitter: 29 mas RMS for the LGSF location and 18mas RMS for the AO system location).

\section{LGSF conceptual design}

The optical schematic of the LGSF is presented in Figure 2. It includes all of the optical sub-systems of the LGSF.

The laser system consists of up to nine $25 \mathrm{~W}$ CW lasers; six are required at first light, eight are required to generate the MOAO asterism and the design allows for one hot spare laser to allow continued AO operation without impacting the AO performance. The lasers are mounted within the azimuth structure to operate in a fixed gravity orientation.

At the output of the lasers, the beams are formatted into a compact $3 \times 3$ configuration and then directed up to the -X Nasmyth platform at a location beneath the telescope elevation axis. From there, the beams are transferred across to the $-\mathrm{X}$ elevation journal along the telescope elevation axis via two active steering mirror arrays and a fold array. The active arrays are used to follow the rotation of the telescope elevation structure as the zenith angle changes, direct the beams in the beamlines 
Fig. 3. LGSF Top End

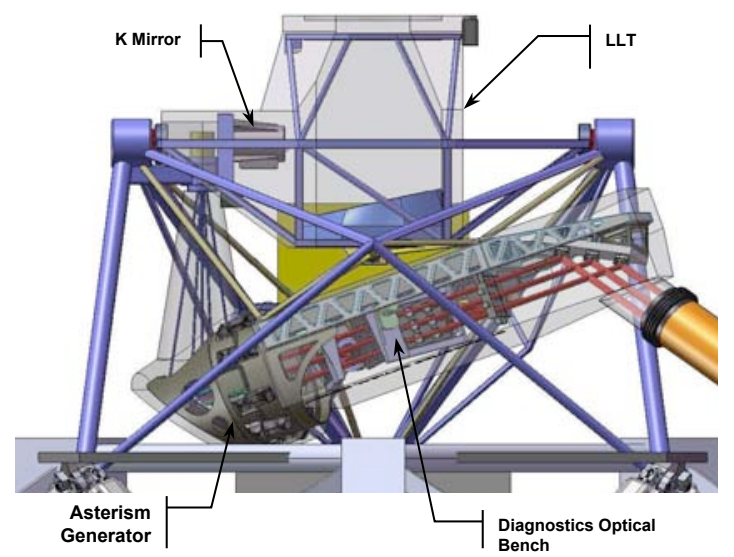

appropriate for each asterism, and to correct for any misalignments of the telescope top end due to thermal and flexure effects. The section of the optical path located along the elevation axis is flexible to allow the telescope to move in elevation, and is retractable to enable seeing-limited instruments to be mounted on the telescope elevation axis. Following the deployable path, the beams are directed along the elevation journal, reflected up along the vertical column of the telescope elevation structure, then up the connecting tripod leg and finally folded to enter the LGSF Top End. Three lenses located along the vertical column path are used to re-image the laser beams from the laser output port onto the entrance pupil of the Laser Launch Telescope.

The LGSF Top End includes (i) the diagnostic system, which monitors beam alignment with respect to telescope top end as well as beam power, (ii) the asterism generator, which transforms the square $3 \times 3$ pattern of beams into the requested asterism, (iii) the $\mathrm{K}$ mirror, which acts as an image rotator and maintains a fixed asterism in the LGS wavefront sensor focal plane and (iv) the Laser Launch Telescope (LLT), which projects the beams to the sky. The LLT is (currently) a $0.5 \mathrm{~m}$ off-axis reflective telescope and is installed on a pivot mount to allow first-order correction of the pointing errors due to telescope top end flexure. The LGSF Top End is illustrated in Figure 3.

The length of the optical path from the laser system to the LGSF Top End is $\sim 78 \mathrm{~m}$. The current design utilizes 143 actuators, around 50 sensors and yields an overall throughput of 0.75 with the required LGS beam quality.

\section{Acknowledgements}

The authors gratefully acknowledge the support of the TMT partner institutions. They are the Association of Canadian Universities for Research in Astronomy (ACURA), the California Institute of Technology and the University of California. This work was supported as well by the Gordon and Betty Moore Foundation, the Canada Foundation for Innovation, the Ontario Ministry of Research and Innovation, the National Research Council of Canada, The Natural Sciences and Engineering Research Council of Canada, the British Columbia Knowledge Development Fund, the Association of Universities for Research in Astronomy (AURA) and the U.S. National Science Foundation.

\section{References}

1. J. Nelson, "Overall science goals and top level AO requirements for TMT," in Adaptive Optics for Extremely Large Telescopes, Y. Clenét, J.-M. Conan, T. Fusco \& G. Rousset, eds., Paris, 2009.

2. B. Ellerbroek, "Adaptive Optics Systems for the Thirty Meter Telescope," Ibid.

3. G. Herriot, "NFIRAOS - first light adaptive optics system for TMT," Ibid.

4. R. Fugate, "Artifical optical guidestars: prospects for extremely large telescopes," in Extremely Large Telescopes: Which Wavelengths?, SPIE Proc. 6986, T. Andersen, ed., 2008

5. C. D'orgeville, "The Gemini South MCAO laser guide star facility: getting ready for first light" in Adaptive Optics Systems, SPIE Proc. 7015, N. Hubin, C. Max, and P. Wizinowich eds., Marseille 2008.

6. R. Joyce, "The laser Guide Star Facility for the Thirty Meter Telescope" in Advances in Adaptive Optics II, SPIE Proc. 6272, B. Ellerbroek and D. Calia eds., Orlando 2008.

7. C. Boyer, "Update on the TMT laser guide star facility design' in Adaptive Optics Systems, SPIE Proc. 7015, N. Hubin, C. Max, and P. Wizinowich eds., Marseille 2008. 